\title{
RSV versus non-RSV bronchiolitis in infants and young children - the bedside characteristics of one epidemic season
}

\author{
Aleksandra Szczawińska-Popłonyk' ', Paulina Komasińska' , Katarzyna Tąpolska-Jóźwiak', \\ Barbara Więckowska², Anna Bręborowicz ${ }^{1}$ \\ 'Department of Paediatric Pneumonology, Allergology, and Clinical Immunology, Poznan University of Medical Sciences, \\ Poznan, Poland \\ ${ }^{2}$ Department of Computer Science and Statistics, Poznan University of Medical Sciences, Poznan, Poland
}

\section{ABSTRACT}

Introduction: Acute lower respiratory infection (ALRI), comprising both bronchiolitis and pneumonia, is a global leading cause of morbidity and hospital admissions of infants and young children, with respiratory syncytial virus (RSV) being a major viral cause. Guidelines recommend a clinical diagnosis of bronchiolitis but do not advise how to distinguish between different viral aetiologies using clinical, laboratory, and radiological criteria.

Aim of the study: The aim of this study was to provide clinical data that characterise RSV and non-RSV aetiology of bronchiolitis for the purpose of better defining the correlations between the viral aetiology and clinical characteristics as well as the risk factors of severe disease.

Material and methods: We retrospectively reviewed 135 records of 116 infants and young children who were hospitalised due to bronchiolitis during one infectious season. A detailed analysis of patients' medical history, clinical course of bronchiolitis, viral aetiology of infection, basic laboratory tests, as well as radiological findings was performed.

Results: Children with RSV bronchiolitis more frequently demonstrated obstruction of the lower airways manifesting with diminished breath sounds and crackles on auscultation and more severe course of the disease than non-RSV patients, defined as hypoxaemia and desaturation, the need for supplemental oxygen, longer duration of oxygen therapy, and a longer hospitalisation.

Conclusions: Clinical symptoms of obstruction of lower airways are more frequent and more severe in RSV than in non-RSV bronchiolitis. Because basic laboratory tests and chest radiographs are not helpful in distinguishing between RSV and non-RSV bronchiolitis, their use in uncomplicated cases should be limited. Therefore, careful clinical assessment and monitoring of children with bronchiolitis is of utmost importance in predicting the course of ALRI and in the implication of therapeutic decisions.

\section{KEY WORDS:}

children, respiratory syncytial virus, acute lower respiratory infection, bronchiolitis.

\section{ADDRESS FOR CORRESPONDENCE:}

Aleksandra Szczawińska-Popłonyk, Department of Paediatric Pneumonology, Allergology, and Clinical Immunology, 27/33 Szpitalna St., 60-572 Poznan, Poland, ORCID: 0000-0001-7244-1882,

e-mail: ola@malwa.com.pl 


\section{INTRODUCTION}

Bronchiolitis is a global leading cause of morbidity and hospital admissions of children under the age of five years. In infants, bronchiolitis is diagnosed in about $70 \%$ of acute lower respiratory infections (ALRI), with respiratory syncytial virus (RSV) being a major viral cause, identified in $76 \%$ [1] to more than $80 \%$ of cases [2]. Among other viral aetiologies in infantile bronchiolitis, human rhinovirus (hRV), influenza virus, coronavirus (hCoV), human metapneumovirus (hMPV), parainfluenza virus (PIV), and human bocavirus (hBoV) have been identified with different frequency due to seasonality, settings, and patient-related demographic data $[1,3]$. The diagnosis of bronchiolitis and the assessment of disease severity is based on patient history and a physical examination [4]. The course of bronchiolitis usually begins with a prodrome of airway infection with rhinorrhoea, low-grade fever, and cough, progressing to tachypnoea, crackles and wheezes on chest examination, and variable degree of respiratory distress with grunting, chest retraction, indrawing, and nasal flaring. The severity of viral bronchiolitis is variable and there are no objective markers to predict the progression of the disease. The risk of a severe clinical course of RSV bronchiolitis in children below two years old is first of all determined by comorbidities, such as chronic lung disease, e.g. cystic fibrosis, haemodynamically significant congenital heart disease, primary or secondary immunodeficiency, neuromuscular disease, trisomy 21, inborn errors of metabolism, and prematurity $[4,5]$. However, the majority of children hospitalised due to RSV-bronchiolitis are previously healthy without any underlying medical condition $[6,7]$, and in these children individual and socioeconomic factors, such as age $<6$ months, siblings, day-care attendance, RSV seasonality, as well as exposure to tobacco smoke and maternal smoking during pregnancy are determinants of disease severity [6]. Moreover, clinical studies have also underlined the role of different respiratory viruses as determinants of clinical course of bronchiolitis assessed by oxygen saturation at admission, need of oxygen supplementation, daily clinical score, and length of hospitalisation, with RSV being the most frequently predominating pathogen responsible for an early first episode of bronchiolitis in young infants $[8,9]$. However, only a few studies have focused on the comparison of laboratory parameters and radiological features of RSV and non-RSV bronchiolitis in hospitalised infants $[5,6,8,10]$.

The aim of the study was to provide clinical data that characterise RSV and non-RSV aetiology of infantile bronchiolitis for the purpose of better defining correlations between the viral aetiology and clinical characteristics and risk factors associated with severe clinical expression. The purpose of the study also had a practical aspect: we wanted to find out if there are typical signs and symptoms discriminating RSV and non-RSV aetiol- ogy of bronchiolitis, which could be helpful for clinicians to make appropriate decisions.

\section{MATERIAL AND METHODS}

\section{STUDY GROUP}

We retrospectively reviewed the medical files of 116 infants and young children, aged from two to 60 weeks, who had been hospitalised due to bronchiolitis in a pneumonology, allergology, and clinical immunology unit of the paediatric university hospital during one season (from September to April). According to the result of an RSV examination in the nasopharyngeal aspirate, the study population was divided into children with RSV(+) and RSV(-) bronchiolitis.

\section{METHODS}

The diagnosis of bronchiolitis in the patients studied was established by an experienced team consisting of paediatric residents, paediatricians, and a paediatric pulmonologist, according to the guidelines of the American Academy of Pediatrics, which defines bronchiolitis as a clinical syndrome assessed by history and physical examination [4]. Based on patients' medical files, we obtained epidemiologic and demographic data, such as the patients' age, gender, prenatal and postnatal history, as well as underlying medical conditions. A clinical assessment of disease severity was performed based on clinical data, the results of physical examination, the need for supplemental oxygen therapy, duration of the hospitalisation, clinical deterioration with progression to respiratory and circulatory insufficiency, and intensive care unit admission.

Basic laboratory test done on admission comprised capillary blood gases, complete peripheral blood count with a differential white cell count, C-reactive protein (CRP), and serum sodium and potassium levels. Detection of RSV in the nasopharyngeal aspirate was performed within the initial 24 hours of hospitalisation using direct immunofluorescence test (IF), and if negative but with strong clinical suspicion of RSV aetiology (as in five cases), IF was verified using RT-PCR (real time-polymerase chain reaction) method.

According to the hospital protocol, in every hospitalised patient with bronchiolitis, a chest X-ray (CXR) was carried out in the emergency room on admission and alternatively during hospitalisation on severe clinical deterioration, and thus coexisting pneumonia was assessed on the basis of radiographic findings, such as consolidations and alveolar infiltrates [11].

\section{STATISTICAL ANALYSIS}

Since the evaluation of differences in aetiology and clinical presentation between the first and the second ep- 


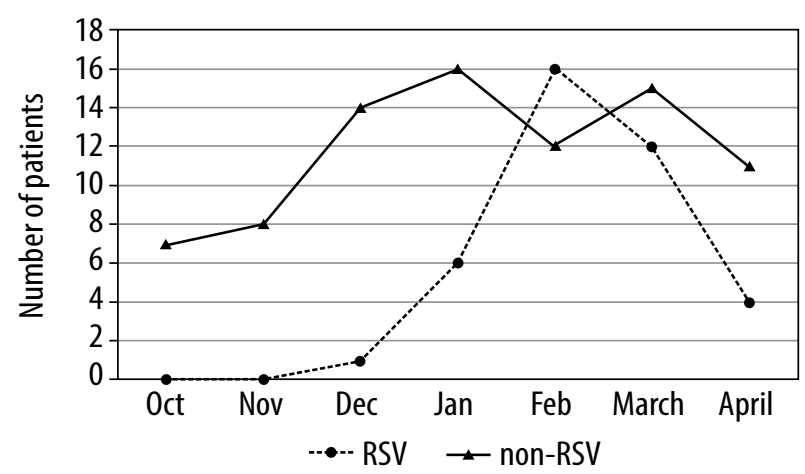

FIGURE 1. Monthly distribution of RSV vs. non-RSV acute lower respiratory infection in the children studied

isode was not the aim of the study, every episode of bronchiolitis was studied separately. All pairwise comparisons between the RSV positive group and non-RSV group were performed using the Mann-Whitney test for continuous variables, the odds ratio (OR), and corresponding $95 \%$ confidence intervals (95\% CI), together with the $\chi^{2}$ or Fisher's exact test, if appropriate, for categorical variables. Logistic regression was used to describe the associations between clinical, laboratory, and radiological manifestations as well as the occurrence of RSV infection adjusted to known risk factors, such as age and sex (aOR). A two-sided $p$-value $<0.05$ was used to test for statistical significance. All statistical analyses were performed using PQStat v1.6.2 software.

\section{RESULTS}

Demographic, epidemiological, and seasonal differences between RSV and non-RSV infections

There were 135 episodes of bronchiolitis in 116 children studied, among whom 74 (64\%) were male. There were eight children born prematurely between the $24^{\text {th }}$ and $29^{\text {th }}$ week of gestational age (WGA), with chronic lung disease of prematurity, who received palivizumab immunoprophylaxis. Among them, two twin infants developed $\mathrm{RSV}(+)$ and the other six RSV(-) bronchiolitis. Among the total 135 episodes of bronchiolitis, in 45 episodes in our study group (33\%), we found an RSV positive test and in as many as 90 episodes (67\%) RSV test result was found negative. The peak of RSV infections was in February and non-RSV infections predominated in January; a detailed monthly distribution of RSV(+) and RSV(-) infections during the study period is shown in Figure 1.

The mean age of RSV(+) children with bronchiolitis was 10 weeks and that of RSV(-) patients was eight weeks; this age difference was not statistically significant $(p=0.33)$. The male gender predominated in both groups, namely 29 episodes (63\%) of RSV(+) and 59 episodes (64\%) of RSV(-) bronchiolitis occurred in boys; however, male gender in our study was not a predisposing factor for RSV infection (OR $=1$; 95\% CI 0.47-2.11). Among the RSV(+) children studied, the majority of them (59\%) had underlying medical conditions, most frequently hypogammaglobulinaemia and food allergy, but none of these factors significantly increased the risk of RSV infection in the children studied $(p=0.11$, $\mathrm{OR}=2.25$; $95 \%$ CI $0.82-6.13$ and $p=0.65$, OR $=0.81$; $95 \%$ CI $0.32-2.02$, respectively). On the contrary, the majority (56\%) of patients with non-RSV infection were previously healthy. Notwithstanding the children in whom RSV was detected, more frequently (54\% of the RSV [+] patients) had positive family history of atopy (asthma and allergic rhinitis in first-degree relatives) compared to non-RSV patients (in $47 \%$ of the RSV[-] children the family history towards atopy was contributory); however, this difference was not statistically significant $(p=0.42)$.

In our study group, prematurity did not pose an increased risk of RSV infection $(p=0.33)$, but maternal pregnancy complications, such as diabetes, hypertension, thyroid dysfunction, and anaemia were associated with more frequent detection of RSV in their children $(\mathrm{OR}=0.34$; 95\% CI 0.16-0.73).

\section{CLINICAL PRESENTATION OF RSV VS. NON-RSV BRONCHIOLITIS}

The most common symptoms reported by parents were rhinitis, coughing, and poor feeding, which were present in both the RSV(+) and the RSV(-) children studied, whereas fever $>38^{\circ} \mathrm{C}$ was found in a minority of children with RSV(+) and RSV(-) ALRI (18\% and 20\% of all episodes, respectively). None of these symptoms were predictors of RSV infection.

On admission, signs and symptoms of bronchiolitis assessed during physical examination did not discriminate between RSV(+) and RSV(-) bronchiolitis except crackles and decreased breath sounds on auscultation, which were more typical and statistically significant ( $p$-values $<0.05$ and 0.03 , respectively) for RSV infection. Distribution of signs and symptoms manifested by children studied along with results of physical examination are presented in Table 1.

\section{CLINICAL COURSE OF RSV VS. NON-RSV BRONCHIOLITIS}

The median duration of therapy in the hospital overall for all the children studied was nine days. RSV infected patients required longer hospitalisation (the median length of stay was 11 days) than children in the RSV(-) group (the median length of stay was seven days) and $\mathrm{RSV}(+)$ cases were significantly more frequently hospitalised for more than nine days, and this correlation was statistically significant $(p=0.00)$.

Furthermore, $73 \%$ of children with RSV(+) bronchiolitis showed hypoxaemia and desaturation that posed a need for supplemental oxygen therapy, compared to $27 \%$ of non-RSV cases for whom oxygen therapy was 
TABLE 1. Symptoms and signs manifested by children with RSV and non-RSV bronchiolitis and assessed in the physical examination

\begin{tabular}{|c|c|c|c|c|c|c|c|c|}
\hline \multirow[t]{2}{*}{ Symptoms and signs } & \multicolumn{2}{|c|}{ RSV } & \multicolumn{2}{|c|}{ Non-RSV } & \multirow[t]{2}{*}{$p$-value } & \multirow[t]{2}{*}{ OR $(95 \% \mathrm{Cl})$} & \multirow[t]{2}{*}{ a OR $(95 \% \mathrm{Cl})$} & \multirow[t]{2}{*}{$p$-value } \\
\hline & $n$ & $\%$ & $n$ & $\%$ & & & & \\
\hline Rhinitis & 34 & 75 & 68 & 75 & 1.00 & $1.00(0.43-2.30)$ & $1.10(0.47-2.58)$ & 0.82 \\
\hline Cough & 44 & 97 & 80 & 89 & 0.09 & $5.45(0.73-243.76)$ & $4.47(0.54-36.85)$ & 0.16 \\
\hline Fever & 9 & 18 & 16 & 20 & 0.75 & $1.15(0.47-2.87)$ & $0.93(0.36-2.54)$ & 0.93 \\
\hline Wheezing & 5 & 11 & 4 & 4 & 0.16 & $2.66(0.54-14.18)$ & $2.51(0.64-9.93)$ & 0.19 \\
\hline Regurgitation & 1 & 2 & 11 & 12 & 0.06 & $0.16(0.04-1.20)$ & $0.18(0.02-1.46)$ & 0.11 \\
\hline Poor feeding & 19 & 42 & 26 & 29 & 0.12 & $1.80(0.85-3.80)$ & $2.08(0.96-4.53)$ & 0.06 \\
\hline Vomiting & 4 & 9 & 9 & 10 & 1.00 & $0.88(0.19-3.39)$ & $0.77(0.22-2.73)$ & 0.69 \\
\hline Diarrhoea & 1 & 2 & 3 & 3 & 1.00 & $0.66(0.01-8.51)$ & $0.44(0.04-4.6)$ & 0.50 \\
\hline Apnoea & 1 & 2 & 2 & 2 & 1.00 & $1.00(0.02-19.70)$ & $0.95(0.08-10.98)$ & 0.97 \\
\hline Tachypnoea & 27 & 61 & 40 & 43 & 0.09 & $1.88(0.91-3.88)$ & $1.76(0.84-3.67)$ & 0.13 \\
\hline Chest wall retraction & 37 & 82 & 60 & 67 & 0.06 & $2.31(0.96-5.58)$ & $2.10(0.86-5.13)$ & 0.10 \\
\hline $\begin{array}{l}\text { Decreased breath } \\
\text { sounds }\end{array}$ & 30 & 67 & 42 & 47 & 0.03 & $2.28(1.08-4.82)$ & $2.11(0.99-4.49)$ & 0.05 \\
\hline Crackles & 30 & 67 & 30 & 33 & $<0.05$ & $4.00(1.87-8.54)$ & $3.99(1.85-8.60)$ & $<0.05$ \\
\hline Rhonchi & 17 & 38 & 48 & 53 & 0.09 & $0.53(0.26-1.10)$ & $0.53(0.25-1.11)$ & 0.09 \\
\hline Wheezing & 16 & 36 & 34 & 38 & 0.80 & $0.91(0.43-1.91)$ & $0.70(0.31-1.57)$ & 0.39 \\
\hline Rales & 9 & 20 & 20 & 22 & 0.77 & $0.88(0.36-2.11)$ & $0.96(0.39-2.35)$ & 0.92 \\
\hline
\end{tabular}

required. The duration of oxygen supplementation was five and four days for the RSV(+) and RSV(-) cases, respectively, and this difference did not prove to be statistically significant $(p=0.45)$. The clinical severity score did not differ between RSV(+) and RSV(-) premature infants who received palivizumab prophylaxis. Among all 135 episodes of bronchiolitis, two of them, one RSV(+) and one RSV(-), developed respiratory failure severe enough to require admission to the Intensive Care Unit.

\section{LABORATORY FINDINGS}

Hypoxaemia and desaturation in capillary blood gas analysis on admission was assessed significantly more frequently in the RSV(+) cases of the children studied with bronchiolitis than in the RSV(-) cases (for hypoxaemia: OR 2.36, 95\% CI [1.06-5.25] and aOR [OR adjusted for age and gender] 2.76 [1.20-6.35]; for desaturation: OR $3.76,95 \%$ CI [1.72-8.22] and aOR 4.15 [1.85-9.34]).

The most frequent abnormality found in basic laboratory tests was thrombocytosis in the peripheral blood cell count, which was observable in 39\% of patients with $\mathrm{RSV}(+)$ and in $23 \%$ of patients with RSV(-) bronchiolitis. Cellular shifts in the white blood cell differential were found in $82 \%$ and $89 \%$ of RSV(+) and RSV(-) cases studied, respectively, and monocytosis was the most frequently observed abnormality with a similar distribution in both groups $(p=0.39)$. The following cellular deviation was lymphocytosis, which was statistically significantly more frequent in $\operatorname{RSV}(-)$ aetiology $(p=0.04)$. The C-re- active protein (CRP) concentration was increased in 30\% of RSV(+) cases and in 19\% of RSV(-) cases, but this difference was not significant $(p=0.19)$. Likewise, sodium and potassium levels did not discriminate between the $\mathrm{RSV}(+)$ and RSV(-) aetiology in the children studied.

\section{RADIOLOGICAL MANIFESTATIONS}

Only four RSV(+) and three RSV(-) children with bronchiolitis had a CXR reported as normal. The most common radiological findings in both RSV and non-RSV patients were hyperinflation and peribronchial thickening, and none of these radiological patterns were associated with RSV or the non-RSV aetiology of bronchiolitis. In two children, one with $\mathrm{RSV}(+)$ bronchiolitis and the other one with RSV(-) bronchiolitis, a CXR revealed massive inflammatory infiltrations in the lung parenchyma as shown in Figure 2 and 3, respectively, clinically correlating with a severe clinical state and respiratory insufficiency.

\section{DISCUSSION}

In our retrospective study, we analysed demographic, seasonal and epidemiological factors, as well as clinical manifestations of both RSV and non-RSV bronchiolitis in infants and children under the age of two years, with the course of the disease severe enough to require hospitalisation. The aetiology of bronchiolitis in the children studied was RSV infection in only 33\% of them, in contrast to other studies reporting RSV aetiology in as many as 


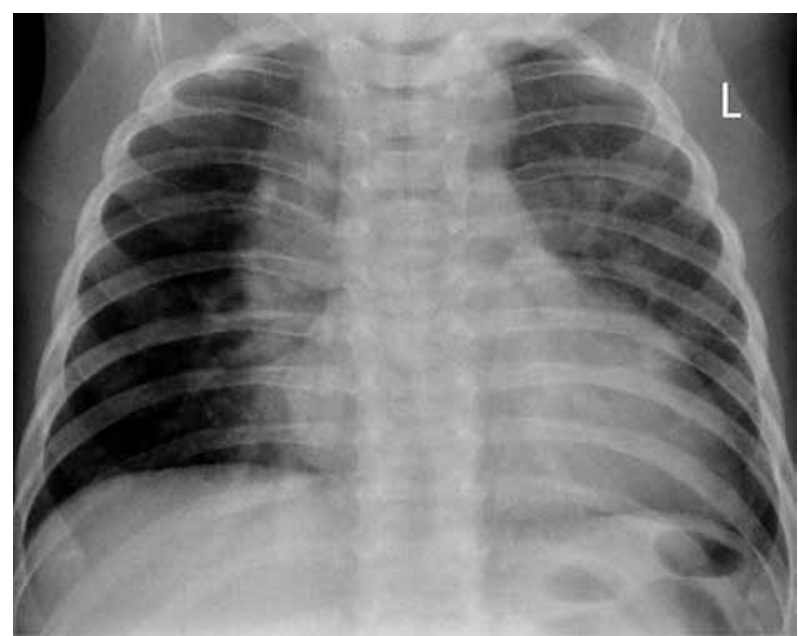

FIGURE 2. Massive pulmonary consolidated parenchymal infiltrations in a four-month-old infant with RSV(+) bronchiolitis

$50-80 \%$ of hospitalised children $<2$ years old with bronchiolitis [1, 10], and ranging widely from $17.5 \%$ [12] to $80.8 \%$ [13] in epidemiological studies that included patients aged from one to 16 years. The practice in our hospital, on admission, is to perform a nasopharyngeal aspirate, and an immunofluorescent test is routinely performed in children to define the aetiology of ALRI; however, its sensitivity and specificity are a matter of debate $[14,15]$ and may be, at least in part, responsible for false negative results. Notwithstanding, in accordance with other clinical and epidemiological studies, the age and gender distribution in our study group showed that $\mathrm{RSV}(+)$ children were most frequently in the 0-3-months age group, with a preponderance of males $[5,16,17]$.

Although prematurity is an important risk factor for RSV bronchiolitis due to impaired innate and adaptive immunity, immune dysregulation with excessive immune cell activation and tissue damage as well as chronic lung disease of prematurity $[18,19]$, in our study prematurity was not associated with an increased risk of an RSV infection. We observed a higher prevalence of prematurity between the $24^{\text {th }}$ and $29^{\text {th }}$ week of gestational age in patients with non-RSV infection, in accordance with the report of Garcia et al. [6]. This effect may be related to anti-RSV immunoprophylaxis with palivizumab. However, it is worth noting that the prophylaxis guidelines are restricted to infants born before the $29^{\text {th }}$ week of gestational age, infants with chronic lung disease of prematurity, and infants with haemodynamically significant heart disease $[20,21]$; thus, preterm infants born at 32-35 weeks of gestational age without these comorbidities, who do not receive anti-RSV prophylaxis, have a substantially higher risk of RSV infection [22-24].

The most common clinical presentation of bronchiolitis in the children studied before admission was rhinorrhoea, coughing, and poor feeding. Coughing and poor feeding were more frequently observed in the RSV $(+)$ ALRI group, but the difference between RSV(+) and

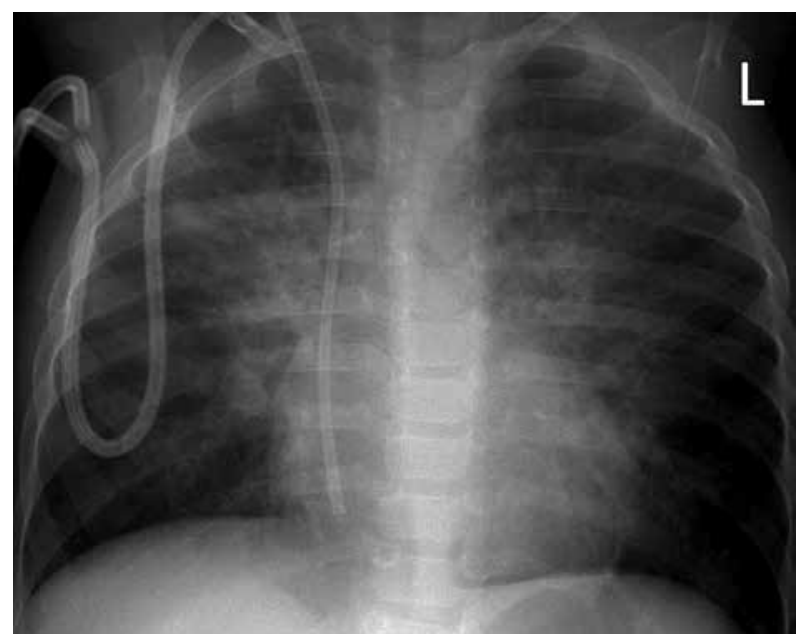

FIGURE 3. RSV(-) bronchiolitis in an 11-month-old infant with associated bilateral inflammatory infiltrations in the central lung zones

RSV(-) groups was not significant. The same conclusions were drawn in the comparable analysis of $40 \mathrm{RSV}$ vs. 40 non-RSV cases reported by Ramagopal et al. [5] and by Manoha et al. [25], based on an analysis of symptomatology of hMPV, hRV, and RSV infections in children under three years old. Poor feeding, in turn, correlating with more severe initial presentation, and the RSV infection in infants with ALRI was observable in the multi-centre, prospective study by Bamberger et al. [26]. In our study, the initial clinical presentation seen on admission to the hospital was more severe in the RSV(+) group of children, with a higher, but not statistically significant incidence of tachypnoea, chest wall retraction, diminished breath sounds, and crackles on chest auscultation. The latter showed a correlation with RSV aetiology in our study group, whereas in other clinical reports, a relationship between wheezes on auscultation and RSV aetiology of bronchiolitis is stressed $[2,5,7,11,27,28]$. A reduced occurrence of wheezes on auscultation in infants with severe presentation of ALRI may be explained by the frequent administration of a nebulised bronchodilator in the emergency department, which may in turn contribute to a partial alleviation of small airway obstruction and to reducing the frequency of wheezing during clinical examination.

In our study, an assessment of clinical disease severity based on a blood gases analysis showed that desaturation and hypoxaemia as well as a requirement for supplemental oxygen and longer duration of oxygen therapy were strongly related to an RSV aetiology of bronchiolitis, and this finding is consistent with reports of other authors [29]. Basic laboratory tests done frequently on admission to the hospital, which serve as a clinical assessment and as the basis for making decisions about general management, such as a complete blood count, a differential white blood cell count, CRP, and electrolytes, did not differ between RSV(+) and RSV(-) groups, as was also shown in other reports [29], and therefore they are generally unhelpful and not to be recommended $[4,29]$. 
In all the children studied, chest radiographs were done in the emergency department, predominantly showing hyperinflation and peribronchial opacities, with similar frequency in both the RSV and non-RSV groups, and therefore neither was it helpful in discriminating between different aetiologies nor did it lead to any implications for clinical management. According to the American Academy of Paediatrics guidelines [4], the CXR routine is not recommended in children with a clinical diagnosis of bronchiolitis because it is a poor indicator of aetiology and only partially contributes to decisions related to treatment modifications [5]. However, it is indicated that, when there is lack of improvement in a hospitalised child at the expected rate or when the clinical course of the disease is severe $[11,30]$, possible pulmonary complications and congenital malformations of the tracheobronchial tree should be excluded. Careful clinical monitoring should be provided for young children with bronchiolitis instead of CXR in uncomplicated cases.

In conclusion, there were no significant differences in clinical symptomatology between RSV and non-RSV aetiology of ALRI before admission. Basic laboratory tests and chest radiographs were not helpful in distinguishing between RSV( $(-)$ and RSV(+) bronchiolitis, and because they are poor indicators of aetiology their use in uncomplicated cases should be limited. In hospitalised children with bronchiolitis, and with results of a physical examination with crackles found on lung auscultation and a more severe clinical course of the disease defined as hypoxaemia and desaturation, the requirement for supplemental oxygen, a longer duration of oxygen therapy, and a longer hospital stay correlated with RSV aetiology. Therefore, the careful clinical assessment and monitoring of children with RSV bronchiolitis is of utmost importance in predicting the course of bronchiolitis and in the implication of therapeutic decisions.

\section{DISCLOSURE}

The authors declare no conflict of interest.

\section{REFERENCES}

1. Miller KE, Gebretsadik T, Carroll KN, et al. Viral etiologies of infant bronchiolitis, croup, and upper respiratory illness during four consecutive years. Pediatr Infect Dis J 2013: 32: 950-955.

2. Piedimonte G, Perez MK. Respiratory syncytial virus infection and bronchiolitis. Pediatr Rev 2014; 35: 519-530.

3. Cangiano G, Nenna R, Frassanito A, et al. Bronchiolitis: analysis of 10 consecutive epidemic seasons. Pediatr Pulmonol 2016; 51: 1330-1335.

4. Raiston SL, Lieberthal AS, Meissner HC, et al. Clinical practice guideline: the diagnosis, management, and prevention of bronchiolitis. Pediatrics 2016; 134: e1474-e1502.

5. Ramagopal G, Brow E, Mannu A, et al. Demographic, clinical and hematological profile of children with bronchiolitis: a comparative study between respiratory syncytial virus (RSV) and non (RSV) groups. J Clin Diagn Res 2016; 10: SC05-SC08.
6. Garcia CG, Bhore R, Soriano-Fallas A, et al. Risk factors in children hospitalized with RSV bronchiolitis versus non-RSV bronchiolitis. Pediatrics 2010; 126: e1453-1460.

7. Breese Hall C, Weinberg GA, Blumkin AK, et al. Respiratory-syncytial virus-associated hospitalizations among children less than 24 months of age. Pediatrics 2013; 132: e341-348.

8. Marguet $C$, Lubrano $M$, Gueudin $M$, et al. In very young infants severity of acute bronchiolitis depends on carried viruses. PLoS One 2009; 4: e4596.

9. Munywoki PK, Koech DC, Agoti CN, et al. Influence of age, severity of infection, and co-infection on the duration of respiratory syncytial virus (RSV) shedding. Epidemiol Infect 2015; 143: 804-812.

10. Hervas D, Reina J, Yanez A, et al. Epidemiology of hospitalization for acute bronchiolitis in children: differences between RSV and non-RSV bronchiolitis. Eur J Clin Microbiol Infect Dis 2012; 31 : 1975-1981.

11. Berg AS, Inchley CS, Fjaerli HO, et al. Clinical features and inflammatory markers in a pediatric pneumonia: a prospective study. Eur J Pediatr 2017; 176: 629-638.

12. Bont L, Checchia PA, Fauroux B, et al. Defining the epidemiology and burden of severe respiratory syncytial virus infection among infants and children in western countries. Infect Dis Ther 2016; 5: 271-298.

13. El Hajje MJ, Lambe C, Moulin F, et al. The burden of respiratory viral disease in hospitalized children in Paris. Eur J Pediatr 2008; 167: 435-436.

14. Moesker FM, van Kampen JJ, Aron G, et al. Diagnostic performance of influenza viruses and RSV rapid antigen detection tests in children in tertiary care. J Clin Virol 2016; 79: 12-17.

15. Chartrand C, Tremblay N, Renaud C, et al. Diagnostic accuracy of rapid detection tests for respiratory syncytial virus infection: systematic review and meta-analysis. J Clin Microbiol 2015; 53: 3738-3749.

16. Nagayama Y, Tsubaki T, Nakayama S, et al. Gender analysis in acute bronchiolitis due to respiratory syncytial virus. Pediatr Allergy Immunol 2006; 17: 29-36.

17. Hacimustafaoglu M, Celebi S, Bozdemir SE, et al. RSV frequency in children below two years hospitalized for lower respiratory tract infection. Turk J Pediatr 2016; 55: 130-139.

18. Ogra PL. Respiratory syncytial virus: the virus, the disease and the immune response. Paediatr Resp Rev 2004; 5 (Suppl A): s119-126.

19. Smith DK, Seales S, Budzik C. Respiratory syncytial virus bronchiolitis in children. Am Fam Physician 2017; 95: 94-98.

20. Abraha HY, Lanctot KL, Paes B. Risk of respiratory syncytial virus infection in preterm infants: reviewing the need for prevention. Expert Rev Respir Med 2015; 9: 779-799.

21. Simoes EA, Anderson EJ, Wu X, et al. Effects of chronologic age and young children exposure on respiratory syncytial virus disease among US preterm infants born at 32 to 35 weeks gestation. PLoS One 2016; 11: e0166226.

22. Anderson EJ, Carbonell-Estrany X, Blanken M, et al. Burden of severe respiratory virus disease among 33-35 weeks gestational age infants born during multiple respiratory syncytial virus seasons. Pediatr Infect Dis 2017; 36: 160-167.

23. Zuccotti G, Fabiano V. Indications to respiratory syncytial virus immunoprophylaxis in the 29-32 wGA group: is there still room for debating? Ital J Pediatr 2017; 43: 17.

24. Bollani L, Baraldi E, Chirico G, et al. Revised recommendations concerning palivizumab prophylaxis for respiratory syncytial virus (RSV). Ital J Pediatr 2015; 41: 97.

25. Manoha C, Espinosa S, Aho SL, et al. Epidemiological and clinical features of hMPV, RSV and RVs infections in young children. J Clin Virol 2007; 38: 221-226. 
26. Bamberger E, Srugo I, Abu Raya B, et al. What is the clinical relevance of respiratory syncytial virus bronchiolitis?: findings from a multi-center, prospective study. Eur J Clin Microbiol Infect Dis 2012; 31: 3323-3330.

27. Justicia-Grande AJ, Pardo-Seco J, Cebey-Lopez M, et al. Development and validation of a new clinical scale for infants with acute respiratory infection: the ReSVinet Scale. PloS One 2016; 11: e0157665.

28. Dumas O, Mansbach JM, Jartti T, et al. A clustering approach to identify severe bronchiolitis profiles in children. Thorax 2016; 71: 712-718.

29. Friedman JN, Rieder MJ, Walton JM. Bronchiolitis: recommendations for diagnosis, monitoring and management of children one to 24 months of age. Paediatr Child Health 2014; 19: 485-491.

30. Arnoux V, Carsin A, Bosdure E, et al. Chest X-ray and acute bronchiolitis: are these indications decreasing? Arch Pediatr 2017; 24: $10-16$. 